\title{
Total Synthesis of Thapsigargin and Nortrilobolide
}

\section{Category}

Synthesis of Natural

Products and

Potential Drugs

\section{Key words}

thapsigargin

nortrilobolide

pinacol coupling

sesquiterpene

lactone

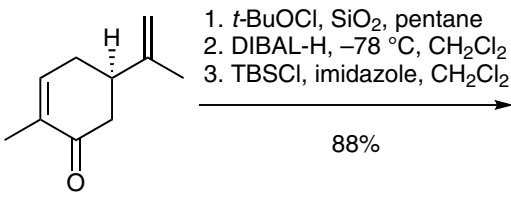

A<smiles>C=C(CCl)[C@H]1CC=C(C)[C@@H](O[Sb])C1</smiles>

B<smiles>COC(=O)C(C)(C)C(=O)COCc1ccccc1</smiles>

$\mathrm{Pd}_{2}(\mathrm{dba})_{3} \cdot \mathrm{CHCl}_{3}$ (S)-BINAP, $\mathrm{LiCl}, 0^{\circ} \mathrm{C}$ THF, then C, LiHMDS

$93 \%$

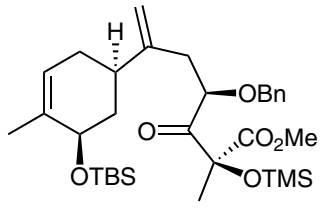

D<smiles>O=C(O)OC1CCCCC1</smiles>

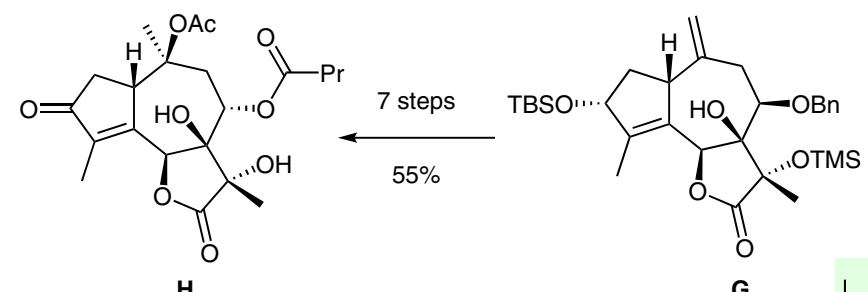

H

$61 \% \downarrow \begin{gathered}\mathrm{Mn}(\mathrm{OAc})_{3}, 80^{\circ} \mathrm{C} \\ \mathrm{PhH}-n-\mathrm{C}_{7} \mathrm{H}_{15} \mathrm{CO}_{2} \mathrm{H}(5: 1)\end{gathered}$
G<smiles>C/C=C(/C)C(=O)OC(=O)c1c(Cl)cc(Cl)cc1Cl</smiles>

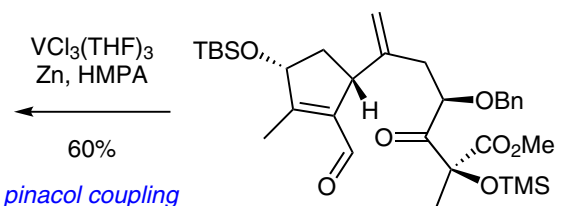

$\mathbf{F}$

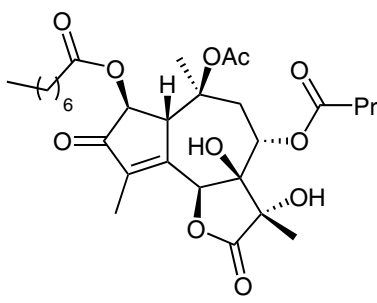

I
1. $\mathrm{Zn}\left(\mathrm{BH}_{4}\right)_{2},-20^{\circ} \mathrm{C}, \mathrm{Et}_{2} \mathrm{O}$ 2. J, $\mathrm{NaHCO}_{3}, 90^{\circ} \mathrm{C}, \mathrm{PhMe}$ $64 \%$

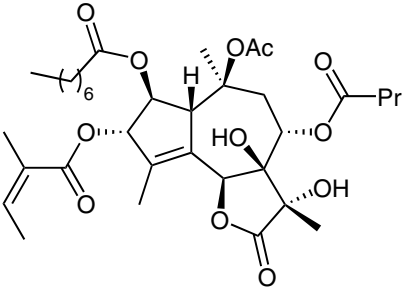

Thapsigargin
Significance: Thapsigargin has attracted great interest over the past 40 years due to its highly oxygenated, complex framework combined with high biological activity. Thapsigargin inhibits intracellular calcium transport at picomolar concentrations. A closely related analogue is currently in phase II clinical trials against liver, brain, prostate, and kidney cancer.
Comment: $(R)-(-)$-Carvone is transformed into $\mathbf{D}$ through allylic chlorination and substitution. An ozonolysis-aldol sequence followed by a pinacol coupling delivers the characteristic 5-7-5 framework in G. Further redox manipulation and sidechain introductions then concisely deliver synthetic thapsigargin. Nortrilobolide lacking the $\alpha$-acyloxy side chain at the ketone was similarly synthesized.

SYNFACTS Contributors: Erick M. Carreira, Philipp Sondermann 